
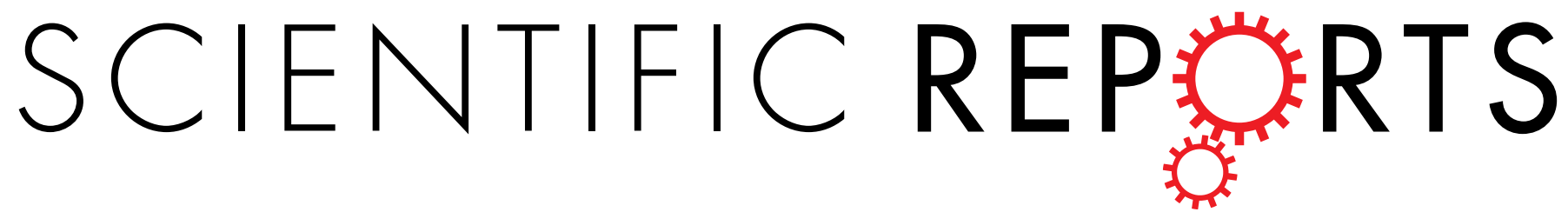

OPEN

Received: 19 February 2015

Accepted: 30 June 2015

Published: 29 July 2015

\title{
Facile and Scalable Preparation of Graphene Oxide-Based Magnetic Hybrids for Fast and Highly
} Efficient Removal of Organic Dyes

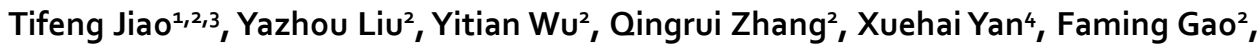
Adam J. P. Bauer ${ }^{3}$, Jianzhao Liu ${ }^{3}$, Tingying Zeng ${ }^{5}$ \& Bingbing $\mathrm{Li}^{3}$

This study reports the facile preparation and the dye removal efficiency of nanohybrids composed of graphene oxide (GO) and $\mathrm{Fe}_{3} \mathrm{O}_{4}$ nanoparticles with various geometrical structures. In comparison to previously reported $\mathrm{GO} / \mathrm{Fe}_{3} \mathrm{O}_{4}$ composites prepared through the one-pot, in situ deposition of $\mathrm{Fe}_{3} \mathrm{O}_{4}$ nanoparticles, the $\mathrm{GO} / \mathrm{Fe}_{3} \mathrm{O}_{4}$ nanohybrids reported here were obtained by taking advantage of the physical affinities between sulfonated $\mathrm{GO}$ and $\mathrm{Fe}_{3} \mathrm{O}_{4}$ nanoparticles, which allows tuning the dimensions and geometries of $\mathrm{Fe}_{3} \mathrm{O}_{4}$ nanoparticles in order to decrease their contact area with $\mathrm{GO}$, while still maintaining the magnetic properties of the nanohybrids for easy separation and adsorbent recycling. Both the as-prepared and regenerated nanohybrids demonstrate a nearly $100 \%$ removal rate for methylene blue and an impressively high removal rate for Rhodamine B. This study provides new insights into the facile and controllable industrial scale fabrication of safe and highly efficient GO-based adsorbents for dye or other organic pollutants in a wide range of environmental-related applications.

Extensive effort has been made to introduce permanently anchored magnetic nanoparticles to graphene oxide (GO) or reduced GO (rGO) sheets ${ }^{1-6}$. For instance, $\mathrm{O}$. Akhavan et al., reported the successful preparation and the magnetic separation application of superparamagnetic $\mathrm{ZnFe}_{2} \mathrm{O}_{4} /$ reduced graphene oxide (rGO) composites by hydrothermal reaction method $^{5}$. In general, magnetic nanocomposites are prepared through the in situ deposition of magnetic nanoparticles (i.e., $\mathrm{Fe}_{3} \mathrm{O}_{4}$ nanoparticles) by co-precipitating iron salts onto $\mathrm{GO} / \mathrm{rGO}$ sheets in aqueous solution ${ }^{1-4}$. Chemically anchoring $\mathrm{Fe}_{3} \mathrm{O}_{4}$ nanoparticles on the $\mathrm{GO} / \mathrm{rGO}$ sheets allows higher loading and uniform distribution of these nanoparticles, giving rise to magnetic composites that possess interesting electrochemical and magnetic properties ${ }^{1-6}$. The $\mathrm{rGO} / \mathrm{Fe}_{3} \mathrm{O}_{4}$ composite containing permanently anchored $\mathrm{Fe}_{3} \mathrm{O}_{4}$ nanoparticles has also been evaluated for its dye adsorption and removal capacity ${ }^{2,3}$. These studies demonstrate the contribution of anchored $\mathrm{Fe}_{3} \mathrm{O}_{4}$ nanoparticles to the composite's magnetic properties, which allow easy magnetic separation of dye-adsorbed composites from water. However, the dye removal capacity of these materials solely depends on the available surface area of GO or rGO sheets (i.e., the available surface area for $\pi-\pi$ stacking interactions) along with abundant functional groups on the GO sheets ${ }^{2,7,8}$. Consequently, the adsorption capacities of pristine $\mathrm{GO}$ or rGO sheets can be compromised due to the permanently anchored $\mathrm{Fe}_{3} \mathrm{O}_{4}$ nanoparticles ${ }^{2,3}$.

${ }^{1}$ State Key Laboratory of Metastable Materials Science and Technology, Yanshan University, Qinhuangdao 066004 , P. R. China. ${ }^{2}$ Hebei Key Laboratory of Applied Chemistry, School of Environmental and Chemical Engineering, Yanshan University, Qinhuangdao 066004, P. R. China. ${ }^{3}$ Department of Chemistry and Biochemistry, Central Michigan University, Mount Pleasant, MI 48859, USA. "National Key Laboratory of Biochemical Engineering, Institute of Process Engineering, Chinese Academy of Sciences, Beijing 100190, P. R. China. ${ }^{5}$ Research Laboratory for Electronics, Massachusetts Institute of Technology, Cambridge, MA 02139, USA. Correspondence and requests for materials should be addressed to Y.W. (email: wu6y@cmich.edu) or B.L. (email: lizb@cmich.edu) 
Particularly, the $\mathrm{Fe}_{3} \mathrm{O}_{4}$ nanoparticles grown through in situ deposition are ultrafine nanoparticles (e.g., less than $50 \mathrm{~nm}$ diameter), which can occupy a greater surface area than relatively larger nanoparticles of the same total mass. To overcome the above drawbacks of the in situ grown, permanently anchored $\mathrm{Fe}_{3} \mathrm{O}_{4}$ nanoparticles, the facile preparation of $\mathrm{GO} / \mathrm{Fe}_{3} \mathrm{O}_{4}$ hybrids reported in this study was achieved by combining $\mathrm{GO}$ and $\mathrm{Fe}_{3} \mathrm{O}_{4}$ nanoparticles through physical affinities between sulfonated $\mathrm{GO}$ and $\mathrm{Fe}_{3} \mathrm{O}_{4}$ nanoparticles. Benefiting from the larger aspect ratio of the GO sheets used in this study, the $\mathrm{Fe}_{3} \mathrm{O}_{4}$ nanoparticles can also be easily wrapped in the GO sheets, further assisting in the magnetic separation. In addition, the $\mathrm{Fe}_{3} \mathrm{O}_{4}$ nanostructures were prepared separately and various dimensions and geometries can be conveniently obtained, allowing us to further explore the effects of the $\mathrm{Fe}_{3} \mathrm{O}_{4}$ nanostructures' dimensions and geometries on the dye adsorption capacity of GO sheets. Overall, the affordable access to $\mathrm{Fe}_{3} \mathrm{O}_{4}$ nanostructures and $\mathrm{GO}$ sheets allow the reported facile preparation procedure to be adopted in various laboratory conditions and to be further optimized for large-scale manufacturing of green and safe dye adsorbents.

\section{Results and Discussion}

In this study, GO nanostructures were prepared according to a modified Hummer's method, ${ }^{9,10}$. The GO sheets were then sulfonated to enhance their surface affinity to $\mathrm{Fe}_{3} \mathrm{O}_{4}$ nanoparticles ${ }^{11,12}$. A typical TEM image of GO nanostructure is shown in Fig. 1A. Magnetic $\mathrm{Fe}_{3} \mathrm{O}_{4}$ nanoparticles with well-defined geometries were prepared separately using a modified solvothermal method ${ }^{13}$. Detailed experimental procedures are included in Methods and are briefly described as follows: sodium hydroxide $(\mathrm{NaOH}$, $0.4 \mathrm{~g}, 0.6 \mathrm{~g}$, or $0.8 \mathrm{~g}$ ) and $1.0 \mathrm{~g}$ of polyethylene glycol $\left(\mathrm{PEG}, \mathrm{M}_{\mathrm{w}} \sim 6000 \mathrm{~g} \cdot \mathrm{mol}^{-1}\right)$ was added to a solution containing $1.35 \mathrm{~g}$ of ferric chloride hexahydrate $\left(\mathrm{FeCl}_{3} \cdot 6 \mathrm{H}_{2} \mathrm{O}\right)$ and $30 \mathrm{~mL}$ of ethylene glycol $\left(\mathrm{C}_{2} \mathrm{H}_{6} \mathrm{O}_{2}\right)$. The above solution mixture was stirred for 30 minutes, transferred into a Teflon-lined stainless steel autoclave $(\sim 100 \mathrm{~mL})$, and then allowed to react for 8 hours at $200^{\circ} \mathrm{C}$. After the reaction was complete, a black precipitate (i.e., $\mathrm{Fe}_{3} \mathrm{O}_{4}$ nanoparticles) was collected with a magnetic block, purified by alternatively washing several times with deionized water and ethanol, and dried at $60^{\circ} \mathrm{C}$ in a vacuum oven. The geometry of the $\mathrm{Fe}_{3} \mathrm{O}_{4}$ nanoparticles was found to vary with the initial mass of $\mathrm{NaOH}$. For instance, when the above synthesis route started with $0.4 \mathrm{~g}$ of $\mathrm{NaOH}$, the as-prepared $\mathrm{Fe}_{3} \mathrm{O}_{4}$ nanoparticles were less than $100 \mathrm{~nm}$ in diameter (Fig. 1B). In contrast, increasing the initial mass of $\mathrm{NaOH}$ to 0.6 g gave rise to nanospheres with a diameter above $100 \mathrm{~nm}$, as shown in Fig. 1C,C'. Further increasing the $\mathrm{NaOH}$ mass to $0.8 \mathrm{~g}$, the collected $\mathrm{Fe}_{3} \mathrm{O}_{4}$ nanoparticles exhibit an interesting multifaceted geometry (Fig. 1D,D'). High magnification TEM images further revealed that the majority of these multifaceted $\mathrm{Fe}_{3} \mathrm{O}_{4}$ nanoparticles are octahedrons (Fig. S1). Figure S2 shows the morphological uniformity of these magnetic nanostructures. The crystalline structure of the as-prepared $\mathrm{Fe}_{3} \mathrm{O}_{4}$ nanoparticles was characterized using X-ray diffraction (Fig. S3) and their magnetic property was evaluated using a vibrating sample magnetometer (VSM, see Fig. S4A).

In comparison to previously reported $\mathrm{GO} / \mathrm{Fe}_{3} \mathrm{O}_{4}$ composites prepared through the in situ deposition of $\mathrm{Fe}_{3} \mathrm{O}_{4}$ nanoparticles, the $\mathrm{GO} / \mathrm{Fe}_{3} \mathrm{O}_{4}$ nanohybrids reported here were obtained through simple yet efficient magnetic separation by taking advantage of the physical affinities between sulfonated GO and $\mathrm{Fe}_{3} \mathrm{O}_{4}$ nanoparticles. The fabrication route is schematically depicted in Fig. 1E (also see Methods). In the experiments, the pre-prepared $\mathrm{Fe}_{3} \mathrm{O}_{4}$ nanoparticles (either 20 or $40 \mathrm{mg}$ ) were dispersed in $40 \mathrm{~mL}$ of $1.25 \mathrm{mg} \cdot \mathrm{mL}^{-1} \mathrm{GO}$ suspension by ultrasonicating at high power. The $\mathrm{GO} / \mathrm{Fe}_{3} \mathrm{O}_{4}$ nanohybrids were collected by magnetic separation to remove free floating GO, if any. The nanohybrid samples thus prepared were designated as G5F2 and G5F4, corresponding to the initial GO/Fe $\mathrm{O}_{4}$ mass ratios of 5:2 and 5:4, respectively. Interestingly, it was found that the amount of free floating GO separated from the magnetic hybrids in a magnetic field was negligible. In addition to the physical affinities between $\mathrm{Fe}_{3} \mathrm{O}_{4}$ nanoparticles and the sulfonated GO sheets, which allow the nanoparticles to settle in-between GO layers, the flexibility and larger aspect ratio of the GO sheets used in this study can also allow $\mathrm{Fe}_{3} \mathrm{O}_{4}$ nanoparticles being wrapped by GO in their suspension, leading to highly efficient magnetic separation. The magnetic property of $\mathrm{GO} / \mathrm{Fe}_{3} \mathrm{O}_{4}$ hybrids was shown in Fig. S4B. Figure 1B, C",D" show the transmission electron micrographs of magnetic $\mathrm{GO} / \mathrm{Fe}_{3} \mathrm{O}_{4}$ nanohybrids prepared from the $\mathrm{Fe}_{3} \mathrm{O}_{4}$ nanoparticles with different geometries, corresponding to Fig. 1B,C,D', respectively.

Raman spectra, as shown in Fig. 2, were further collected to characterize the magnetic composite materials prepared in this study. Raman shift at $1601 \mathrm{~cm}^{-1}$, also called $\mathrm{G}$ band, was attributed to the first-order scattering of the $\mathrm{E}_{2} \mathrm{~g}$ phonons of $\mathrm{sp}^{2}$-hybridized carbon atoms, while Raman shift at $1351 \mathrm{~cm}^{-1}$, i.e., $\mathrm{D}$ band ascribed to the breathing mode of the $\kappa$-point phonons of the $\mathrm{A}_{1} \mathrm{~g}$ symmetry, originated from defects involved in $\mathrm{sp}^{3}$-hybridized carbon bonds (e.g., hydroxyl and epoxide bonds) ${ }^{14,15}$. In addition to the $\mathrm{G}$ and $\mathrm{D}$ bands, a broad peak at $2692 \mathrm{~cm}^{-1}$ (i.e., $2 \mathrm{D}$ band) was also observed. The intensity of $2 \mathrm{D}$ band is correlated to the stacking mode of graphene sheets ${ }^{16}$. Previous study has shown that, for single-layer graphene sheets, the $G$ and $2 \mathrm{D}$ bands appear at 1585 and $2679 \mathrm{~cm}^{-1}$, respectively, while for multi-layer graphene sheets, both the $\mathrm{G}$ and $2 \mathrm{D}$ bands can shift in Raman spectra ${ }^{17,18}$, as shown in this study. Furthermore, the 2D/G intensity ratios for the single and bilayer GO sheets were found in the range of $1.53-1.68$ and $0.82-0.89$, respectively, as previously reported by O. Akhavan et al. ${ }^{19,20}$ Previous studies have also shown that the $2 \mathrm{D} / \mathrm{G}$ intensity ratios for single-, double-, triple-, and multi- $(>4)$ layer graphene sheets are 1.6, 0.8, 0.30 and 0.07 , respectively ${ }^{21,22}$. Figure $2 \mathrm{D}$ shows that the $2 \mathrm{D} / \mathrm{G}$ ratios of the GO sheets and four different composite samples prepared in this study have values between 0.09 and 0.11 , further suggesting the multilayer nature of the GO-based materials. In addition, the G/D peak 


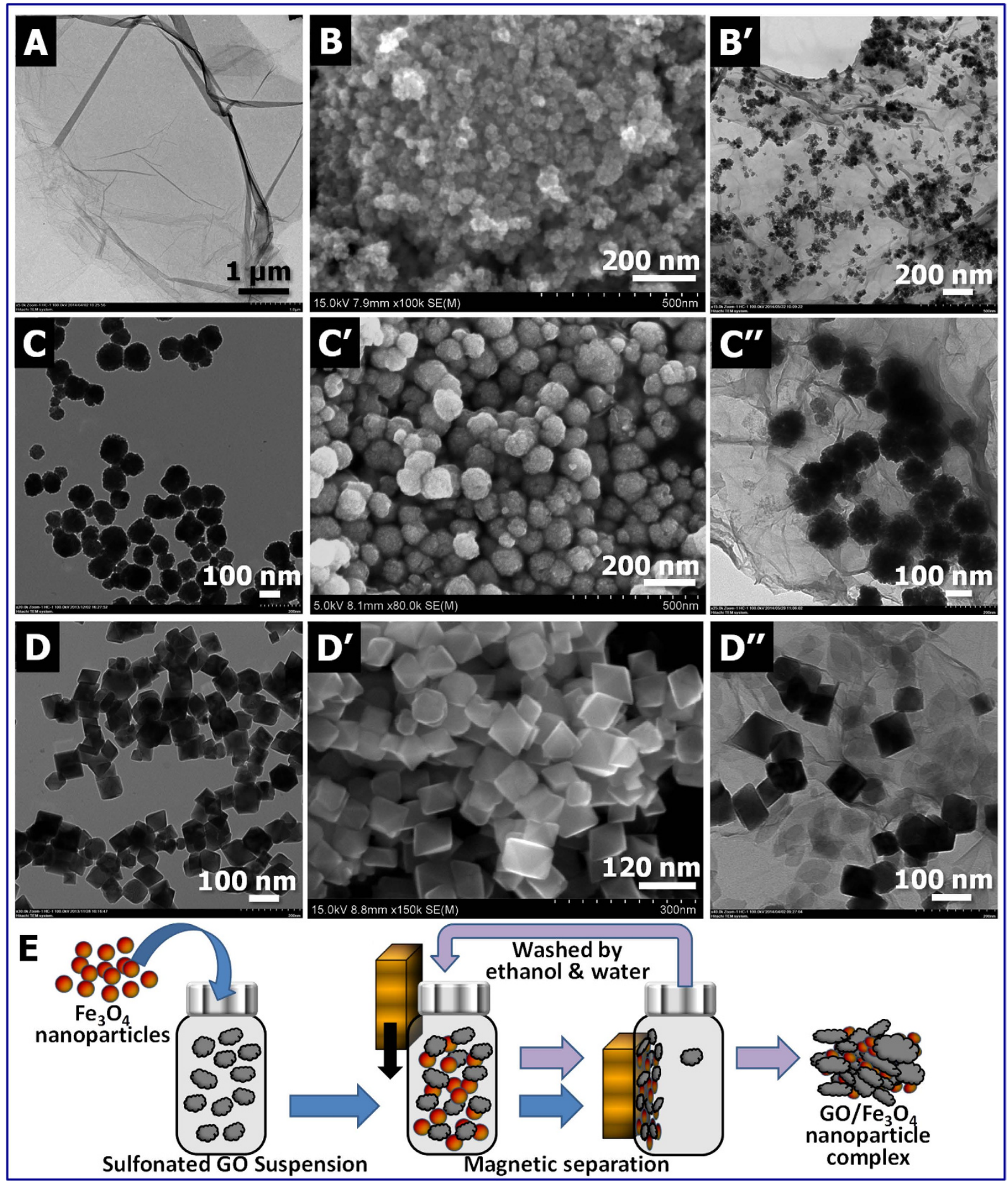

Figure 1. (A) A TEM image of a GO sheet. Morphology of $\mathrm{Fe}_{3} \mathrm{O}_{4}$ nanoparticles includes (B) fine nanoparticles ( $<100 \mathrm{~nm}$ diameter), (C, C') nanospheres ( $100 \mathrm{~nm}$ diameter), and (D, D') multifaceted nanoparticles. The nanohybrids shown in (B', C', and D") were prepared with $\mathrm{Fe}_{3} \mathrm{O}_{4}$ nanoparticles seen in (B, C', and D'), respectively, using 5:4 by mass $\mathrm{GO} / \mathrm{Fe}_{3} \mathrm{O}_{4}$ (i.e., G5F4). [Note: TEM images: A, B', C, C', D, and D', SEM images: B, C' and D'].

intensity ratio, known as a measure of the $\mathrm{sp}^{2}$ domain size of graphene sheets containing $\mathrm{sp}^{3}$ and $\mathrm{sp}^{2}$ bonds, only exhibits a negligible change for the GO-based magnetic composites in comparison with that of the neat GO sample (see Fig. 2C), implying that the property of the GO was not chemically altered during the preparation of the magnetic composites.

In the past years, the one-pot synthesis of GO-based nanocomposites through the in situ growth of nanostructures directly onto GO sheets has been developed into a state-of-art science subject ${ }^{1-6,23,24}$. 

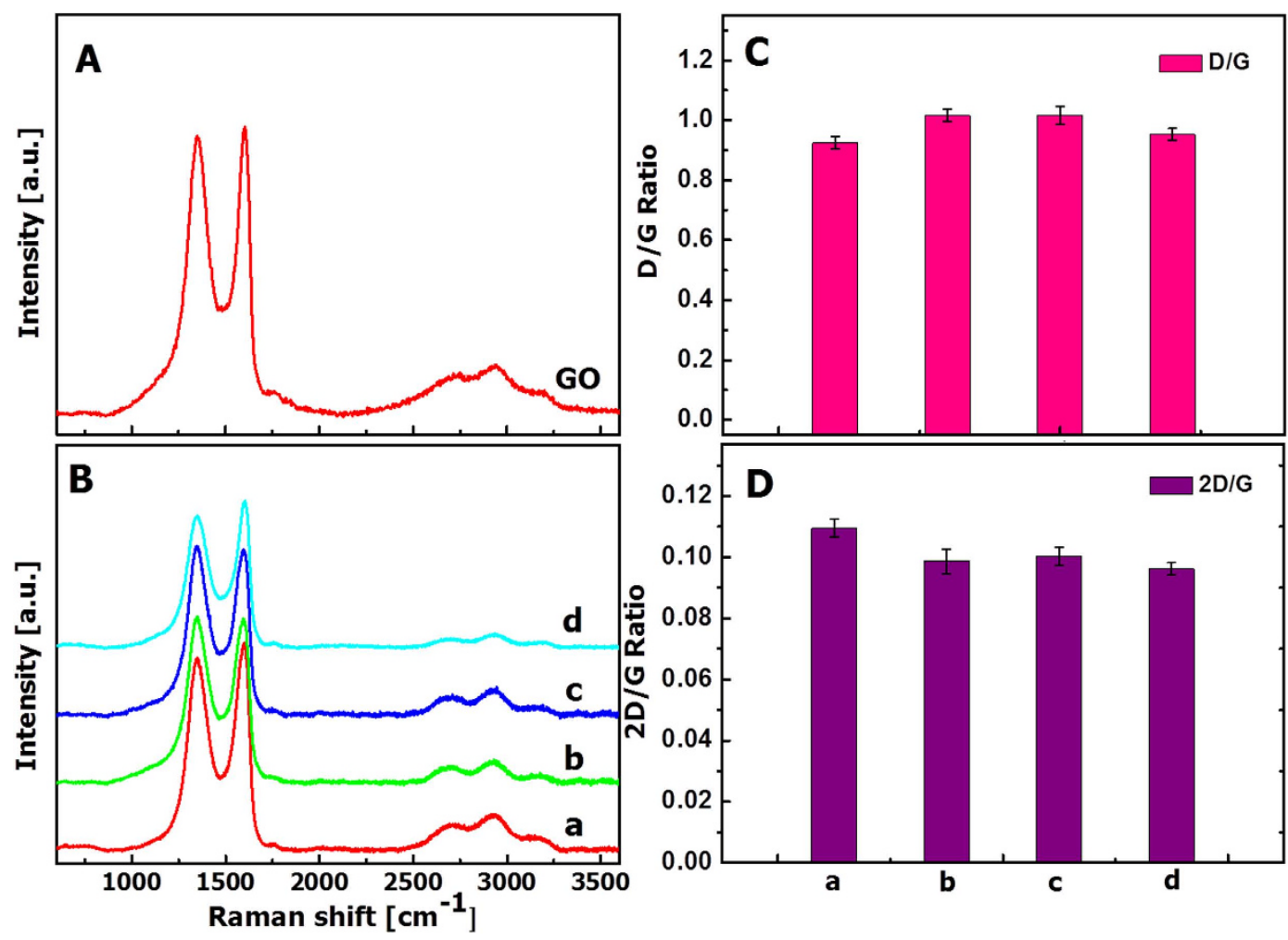

Figure 2. Raman spectra of lyophilized (A) GO and (B) hybrid composites. The intensity ratios of D/G and $2 \mathrm{D} / \mathrm{G}$ in the Raman spectra shown in $(\mathbf{B})$ were plotted in $(\mathbf{C})$ and $(\mathbf{D})$, respectively. [Note: Hybrid composites $(\mathbf{a}, \mathbf{b}, \mathbf{c})$ were prepared with the $\mathrm{Fe}_{3} \mathrm{O}_{4}$ nanoparticles seen in Fig. 1B, C', D', respectively, using 5:4 by mass $\mathrm{GO} / \mathrm{Fe}_{3} \mathrm{O}_{4}$. Hybrid composite (d) was prepared with purchased $\mathrm{Fe}_{3} \mathrm{O}_{4}$ nanoparticles using 5:4 by mass $\mathrm{GO} / \mathrm{Fe}_{3} \mathrm{O}_{4}$.

Some of these GO-based nanocomposites have found their niches in potential applications as energy storage devices ${ }^{25}$, supercapacitors ${ }^{26}$, and electrode materials ${ }^{27}$. However, when the goal of a research task is to remove organic dyes by utilizing the superior adsorption capacity of a GO surface, the available surface area of GO sheets (i.e., the available surface area for $\pi-\pi$ stacking interactions) along with the abundance of functional groups on the GO sheets becomes the most predominant factor in determining the dye removal efficiency. For instance, for $\mathrm{GO} / \mathrm{Fe}_{3} \mathrm{O}_{4}$ nanocomposites containing in situ precipitated ultrafine nanoparticles (e.g., less than $50 \mathrm{~nm}$ diameter), the adsorption efficiency of pristine GO sheets could be decreased because (1) the $\mathrm{Fe}_{3} \mathrm{O}_{4}$ nanoparticles are permanently anchored on the GO sheets and (2) the ultrafine nanoparticles can occupy a greater surface area than relatively larger nanoparticles of the same total mass. Thus, the facile preparation of $\mathrm{GO} / \mathrm{Fe}_{3} \mathrm{O}_{4}$ nanohybrids reported in this study was aimed at overcoming the above drawbacks by (1) combining the $\mathrm{GO}$ and $\mathrm{Fe}_{3} \mathrm{O}_{4}$ nanoparticles through relatively weak physical affinities and (2) tuning the dimensions and geometries of $\mathrm{Fe}_{3} \mathrm{O}_{4}$ nanoparticles to decrease the contact area with GO, while still maintaining the magnetic properties of the nanohybrids for easy separation and adsorbent recycling. The dye removal efficiency of the $\mathrm{GO} / \mathrm{Fe}_{3} \mathrm{O}_{4}$ nanohybrids prepared in our study was evaluated thoroughly and the results are discussed below.

Two cationic dyes, methylene blue (MLB) and Rhodamine B (RhB), and one anionic dye, methyl blue (MB), were used in this study. Their molecular structures, space-filling models, and dimensions are shown in Fig. 3. Figure 4 shows the percent removal rate versus time plots for removing each dye in its aqueous solution $\left(20 \mathrm{mg} \cdot \mathrm{L}^{-1}, 5 \mathrm{~mL}\right)$ using $3 \mathrm{mg}$ of G5F4 nanohybrids prepared from (a) fine nanoparticles, (b) nanospheres, and (c) multifaceted nanoparticles. The percent dye removal rate was probed based on absorption spectroscopy (see Methods). Figure 4A shows an impressively high removal rate for $\mathrm{MLB}$, which reaches above $95 \%$ within only 30 minutes, regardless of the geometry and dimensions of the incorporated $\mathrm{Fe}_{3} \mathrm{O}_{4}$ nanostructures. The adsorption behavior of $\mathrm{RhB}$ is also independent of the type of $\mathrm{Fe}_{3} \mathrm{O}_{4}$ nanostructures, as shown in Fig. 4B. The removal rate for $\mathrm{RhB}$ can also reach above $80 \%$ within just 30 minutes, though the rate is lower than that for MLB. In contrast, the adsorption rate for $\mathrm{MB}$ is found to vary with the dimensions of the $\mathrm{Fe}_{3} \mathrm{O}_{4}$ nanoparticles. For instance, the $\mathrm{MB}$ removal rate using $\mathrm{GO} / \mathrm{Fe}_{3} \mathrm{O}_{4}$ nanohybrids containing finer nanoparticles (see morphology in Fig. 1B,B') is less than $40 \%$ within the first 30 minutes, which is lower than the approximately $50 \%$ achieved by using nanohybrids containing larger nanospheres (see morphology in Fig. 1C-C") and multifaceted nanoparticles (see morphology in Fig. 1D-D"). In addition, for MB solutions, the nanohybrids containing multifaceted 


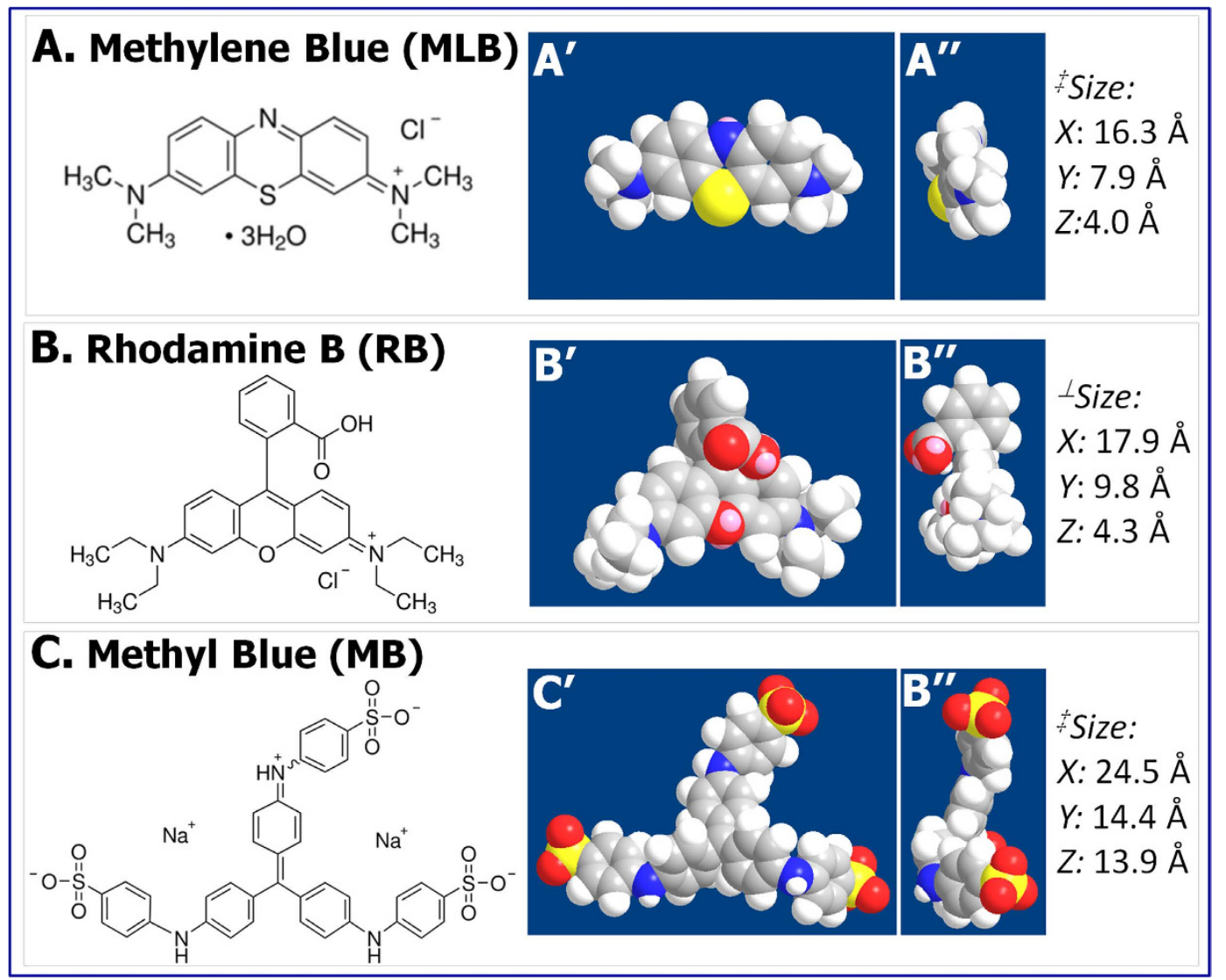

Figure 3. Chemical structures of $(\mathbf{A}) \mathrm{MLB},(\mathbf{B}) \mathrm{RhB}$, and (C) $\mathrm{MB}$, along with the top (A', B', and $\left.\mathbf{C}^{\prime}\right)$ and side (A", B", and C”) view of their space-filling models. [Note: ${ }^{\ddagger}$ Ref. 29 and ${ }^{\perp}$ Ref. 30].

nanoparticles did exhibit a slightly higher dye adsorption capacity than those prepared using larger nanospheres. Nevertheless, the $\mathrm{GO} / \mathrm{Fe}_{3} \mathrm{O}_{4}$ nanohybrids thus prepared possess a much higher adsorption capacity for MLB and $\mathrm{RhB}$ than for MB, which can be attributed to the fact that (1) the electrostatic attractions between GO sheets and cationic dyes (i.e., MLB and RhB) are strong and (2) both the quality and quantity of $\pi-\pi$ stacking interactions could decrease from MLB, to RhB, to MB, as suggested in Fig. 3. In Fig. 3, the side view of the space-filling models implies that the quality of molecular conjugation might decrease from $M L B$, to $\mathrm{RhB}$, to $\mathrm{MB}$, with the increasing dimensions of these dye molecules.

Figure 5 shows the percent dye removal rate versus time plots for the adsorption of (A) MLB, (B) $\mathrm{RhB}$, and (C) $\mathrm{MB}$, using nanohybrids with different $\mathrm{GO}$ to $\mathrm{Fe}_{3} \mathrm{O}_{4}$ mass ratios. [Note: The nanohybrids used here were prepared using the multifaceted nanoparticles, as seen in Fig. 1D,D'.] For MLB, the percent dye removal rate is comparable for both G5F2 and G5F4 samples and is nearly $100 \%$ within 30 minutes. In contrast, for both $\mathrm{RhB}$ and $\mathrm{MB}$, when increasing the $\mathrm{GO}$ content in the nanohybrid samples, the dye adsorption capacity increases significantly, such as for the G5F2 sample, clearly indicating that it is the exposed GO surface area playing the determining role in the adsorption capacity of the $\mathrm{GO} / \mathrm{Fe}_{3} \mathrm{O}_{4}$ nanohybrids. The above observation is in agreement with that found in $\mathrm{rGO} / \mathrm{Fe}_{3} \mathrm{O}_{4}$ composites containing chemically anchored $\mathrm{Fe}_{3} \mathrm{O}_{4}$ nanoparticles ${ }^{2}$. For G5F4 samples prepared using multifaceted nanoparticles (Fig. 1C,C'), the adsorption kinetics were further evaluated by fitting experimental data using a pseudo-second-order adsorption equation: $t / q_{t}=1 /\left[k_{2} q_{e}^{2}\right]+t / q_{e}$, where $t$ is the adsorption time, $q_{e}$ is the adsorption capacity at equilibrium, $k_{2}$ is the pseudo-second-order rate constant, and $q_{t}$ is the adsorption capacity at time $t^{28}$. The fitting results are summarized in a table in Fig. 6 . In comparison to previous reports on $\mathrm{RhB}$ adsorption using $\mathrm{GO}$ or $\mathrm{rGO}$-based composites with in situ deposited $\mathrm{Fe}_{3} \mathrm{O}_{4}$ nanoparti$\mathrm{cles}^{2,3}$, the $q_{e}$ value of $30 \mathrm{mg} \cdot \mathrm{g}^{-1}$ is comparable to the reported values. Furthermore, the kinetic adsorption experiments using the G5F2 samples gave rise to an even greater $q_{e}$ value of $32 \mathrm{mg} \cdot \mathrm{g}^{-1}$. It is also worth mentioning that, in this study, both the compositions of the $\mathrm{GO} / \mathrm{Fe}_{3} \mathrm{O}_{4}$ nanohybrids and other experimental conditions (e.g., pH, temperature, adsorption time, etc.) are not optimized for large scale applications. It is therefore reasonable to expect even higher $q_{e}$ values after optimizing those experimental parameters that can potentially affect the adsorption capacity of the $\mathrm{GO} / \mathrm{Fe}_{3} \mathrm{O}_{4}$ nanohybrids.

Extensive effort has been made to introduce permanently anchored $\mathrm{Fe}_{3} \mathrm{O}_{4}$ nanoparticles to $\mathrm{GO}$ or rGO sheets. The main goal of incorporating magnetic $\mathrm{Fe}_{3} \mathrm{O}_{4}$ nanoparticles in these studies, if not the 


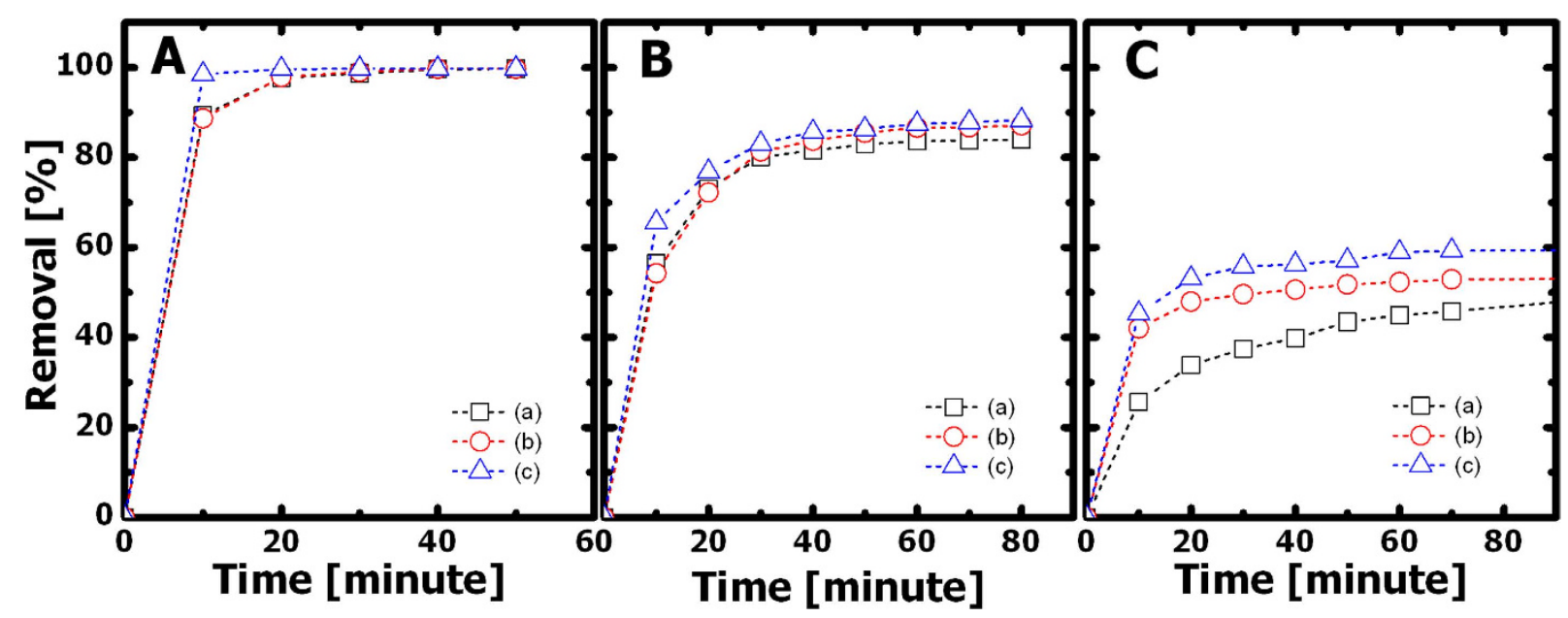

Figure 4. The percent dye removal rate versus time plots for the adsorption of $(A) M L B,(B) R h B$, and (C) $\mathrm{MB}$ using $\mathrm{GO} / \mathrm{Fe}_{3} \mathrm{O}_{4}$ nanohybrids (G5F4) prepared from (a) fine nanoparticles, (b) nanospheres, and (c) multifaceted nanoparticles.

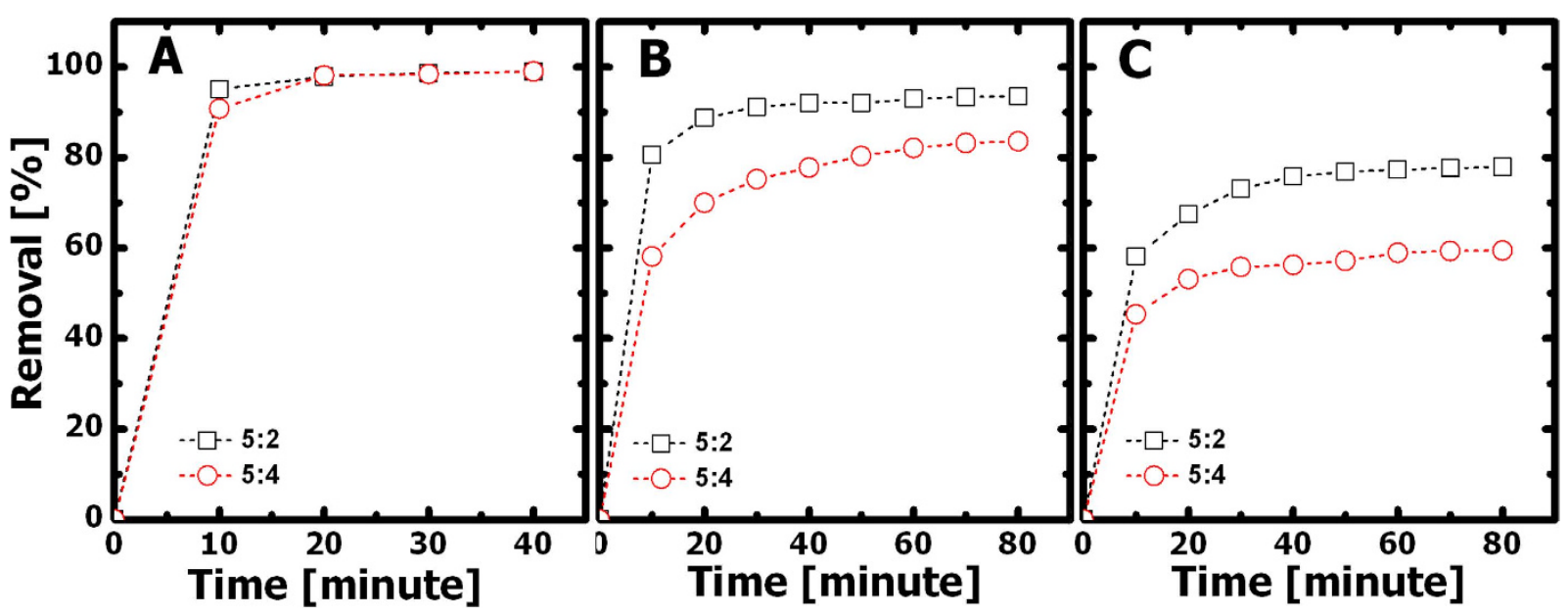

Figure 5. The percent dye removal rate versus time plots for the adsorption of (A) MLB, (B) RhB, and (C) $\mathrm{MB}$ using nanohybrids prepared with initial $\mathrm{GO} / \mathrm{Fe}_{3} \mathrm{O}_{4}$ mass ratios of 5:2 (G5F2) and 5:4 (G5F4), respectively.

only one, is to achieve rapid magnetic separation of dye-adsorbed composites from a water environment. However, as mentioned early, the functionalities and the available surface area of GO sheets are consequently compromised because of the anchored $\mathrm{Fe}_{3} \mathrm{O}_{4}$ nanoparticles. Moreover, it is fairly difficult to precisely control the composition of the final products synthesized through the one-pot, in situ deposition of $\mathrm{Fe}_{3} \mathrm{O}_{4}$ nanoparticles. This difficulty in compositional control could also prevent further large-scale industrial applications of these composites as adsorbents for dye or other organic pollutants. In sharp contrast, the $\mathrm{Fe}_{3} \mathrm{O}_{4}$ nanoparticles introduced in this study were only physically attached onto GO sheets and served mainly as magnetic agents for rapidly extracting dye-adsorbed GO sheets from an aqueous environment. Benefiting from both the rapid yet efficient magnetic separation and the weak affinities between sulfonated $\mathrm{GO}$ and $\mathrm{Fe}_{3} \mathrm{O}_{4}$ nanoparticles, the hybrid materials reported here can be easily regenerated.

In experiments, the percent dye removal rate acquired using an as-prepared G5F4 sample was determined after 12-hour adsorption with constant shaking and the value was designated as Round 1 (Fig. 7). The dye-adsorbed hybrid magnetically extracted from the aqueous solution was washed alternatively using ethylene glycol and ethanol several times, then washed with DI water, and finally, freeze-dried in a lyophilizer. It is also worth noting that the organic washing solvent turned to color solutions immediately after the dye molecules were released from the $\mathrm{GO} / \mathrm{Fe}_{3} \mathrm{O}_{4}$ nanohybrids. This observation further suggests that the removal of organic dyes from aqueous solutions was achieved through the adsorption capacity of the prepared hybrid materials, which was also evidenced by the fitting results based on the 


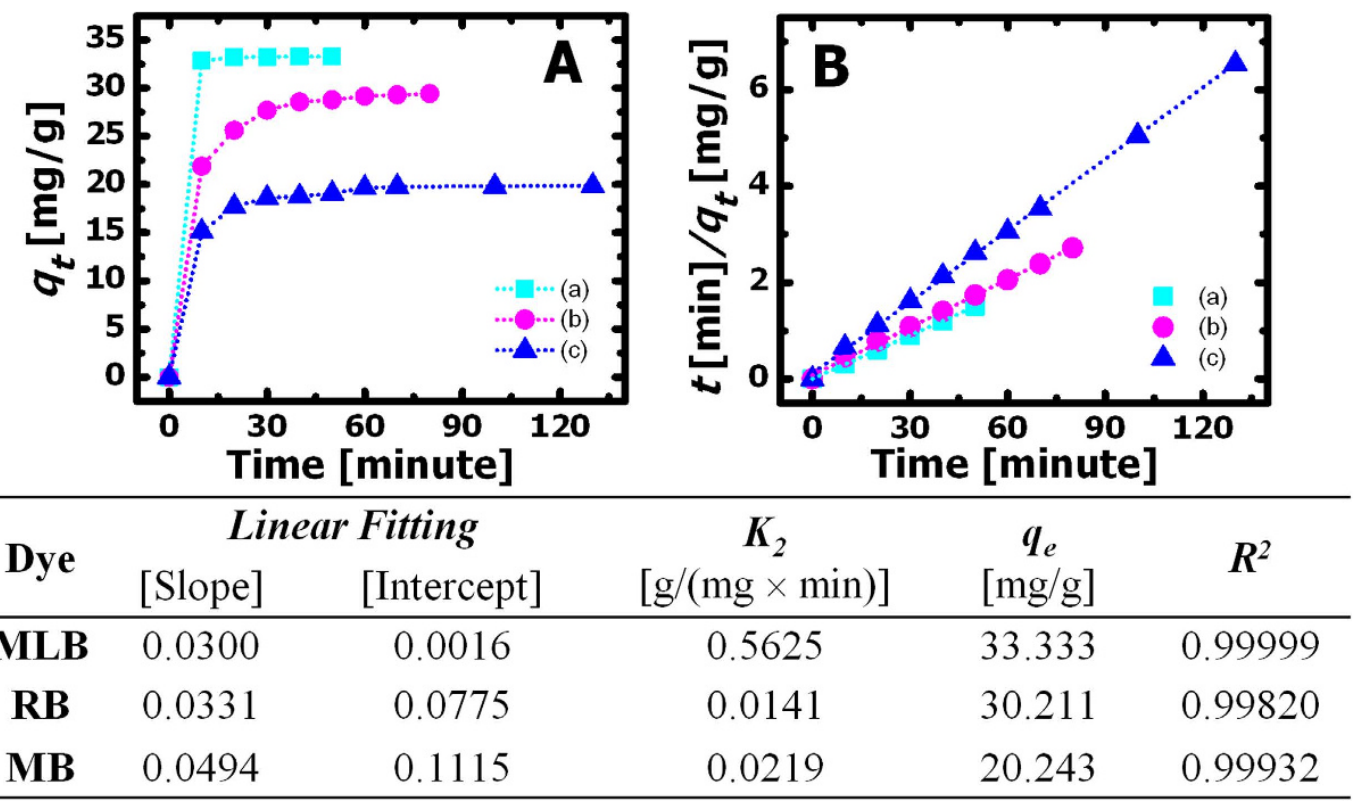

Figure 6. Kinetic adsorption (A) $q_{t}$ versus $t$ plots and (B) $t / q_{t}$ versus $t$ plots for (a) MLB, (b) RhB, and (c) MB. The table lists the fitting results achieved for kinetic adsorption data using the pseudo-second-order adsorption equation.

pseudo-second-order adsorption kinetics, as discussed above. In addition, the $\mathrm{Fe}_{3} \mathrm{O}_{4}$ nanoparticles prepared here do not exhibit a photocatalytic effect on the degradation/removal of the three dyes (Fig. S5, S6, and S7), further demonstrating that the removal of the organic dyes discussed in this study is due to the high adsorption capacity of the $\mathrm{GO} / \mathrm{Fe}_{3} \mathrm{O}_{4}$ hybrid composites. The regenerated hybrid composites were also used in the subsequent adsorption experiment, following the same procedure adopted in the $1^{\text {st }}$ Round experiment. The percent dye removal rate then determined was reported as the $2^{\text {nd }}$ Round result. For cationic MLB, the removal rate can reach nearly $100 \%$ using regenerated $\mathrm{GO} / \mathrm{Fe}_{3} \mathrm{O}_{4}$ nanohybrids even after the $6^{\text {th }}$ Round (Fig. 7A), indicating that the recycling process hardly sacrifices the adsorption capacity of the hybrids for the MLB. For both RhB and MB, the percent removal rates decrease after each round of regeneration (Fig. 7B,C), suggesting that optimizing the hybrid compositions, adsorption conditions, and adsorbent recycling procedures is necessary to further improve the potency of regenerated $\mathrm{GO} / \mathrm{Fe}_{3} \mathrm{O}_{4}$ hybrids as adsorbents for $\mathrm{RhB}$ and $\mathrm{MB}$. Nevertheless, the percent dye removal rates for $\mathrm{RhB}$ can reach up to $90 \%$ (the $1^{\text {st }}$ Round) and $80 \%$ (the $2^{\text {nd }}$ round), which are still fairly high in comparison to previously reported values ${ }^{2,3}$.

\section{Conclusions}

This study reports the facile preparation and the dye removal efficiency of $\mathrm{GO} / \mathrm{Fe}_{3} \mathrm{O}_{4}$ nanohybrids. In comparison to previously reported $\mathrm{GO} / \mathrm{Fe}_{3} \mathrm{O}_{4}$ composites prepared through the one-pot, in situ deposition of $\mathrm{Fe}_{3} \mathrm{O}_{4}$ nanoparticles, the $\mathrm{GO} / \mathrm{Fe}_{3} \mathrm{O}_{4}$ nanohybrids reported here were obtained by taking advantage of the physical affinities between sulfonated $\mathrm{GO}$ and $\mathrm{Fe}_{3} \mathrm{O}_{4}$ nanoparticles. More importantly, since the $\mathrm{Fe}_{3} \mathrm{O}_{4}$ nanoparticles were prepared separately in this study, it was therefore possible to tune the dimensions and geometries of $\mathrm{Fe}_{3} \mathrm{O}_{4}$ nanoparticles to decrease their contact area with $\mathrm{GO}$ sheets, while still retaining their magnetic properties for easy separation and adsorbent recycling. For cationic MLB, the percent dye removal rate can reach nearly $100 \%$ by using regenerated $\mathrm{GO} / \mathrm{Fe}_{3} \mathrm{O}_{4}$ hybrids even after the $6^{\text {th }}$ round, indicating the high potency of these hybrids. For cationic RhB, the percent removal rates can reach $90 \%$ in the $1^{\text {st }}$ round, $80 \%$ in the $2^{\text {nd }}$ round, and still above $60 \%$ in the $6^{\text {th }}$ round, which are comparable to or even better than previously reported results. This study provides new insights into the facile and controllable industrial scale fabrication of safe and highly efficient GO-based adsorbents for dye or other organic pollutants in a wide range of environmental-related applications.

\section{Methods}

Materials. Ferric chloride hexahydrate $\left(\mathrm{FeCl}_{3} \cdot 6 \mathrm{H}_{2} \mathrm{O}, 98 \%\right)$, ethylene glycol, and graphite powder (200 mesh) were purchased from Tianjin Kaitong Chemicals (Tianjin, China). Rhodamine B, methylene blue, and methyl blue were obtained from Beijing Chemicals (analytical reagent grade, Beijing, China). All other solvents and reagents used in this study were purchased from Sinopharm Chemical Reagent Co. Ltd (analytical reagent grade, Shanghai, China). The deionized (DI) water was obtained using a Millpore Milli-Q water purification system with a resistivity of $18.2 \mathrm{M} \Omega \mathrm{cm}^{-1}$. All chemicals were used as received without further purification. 


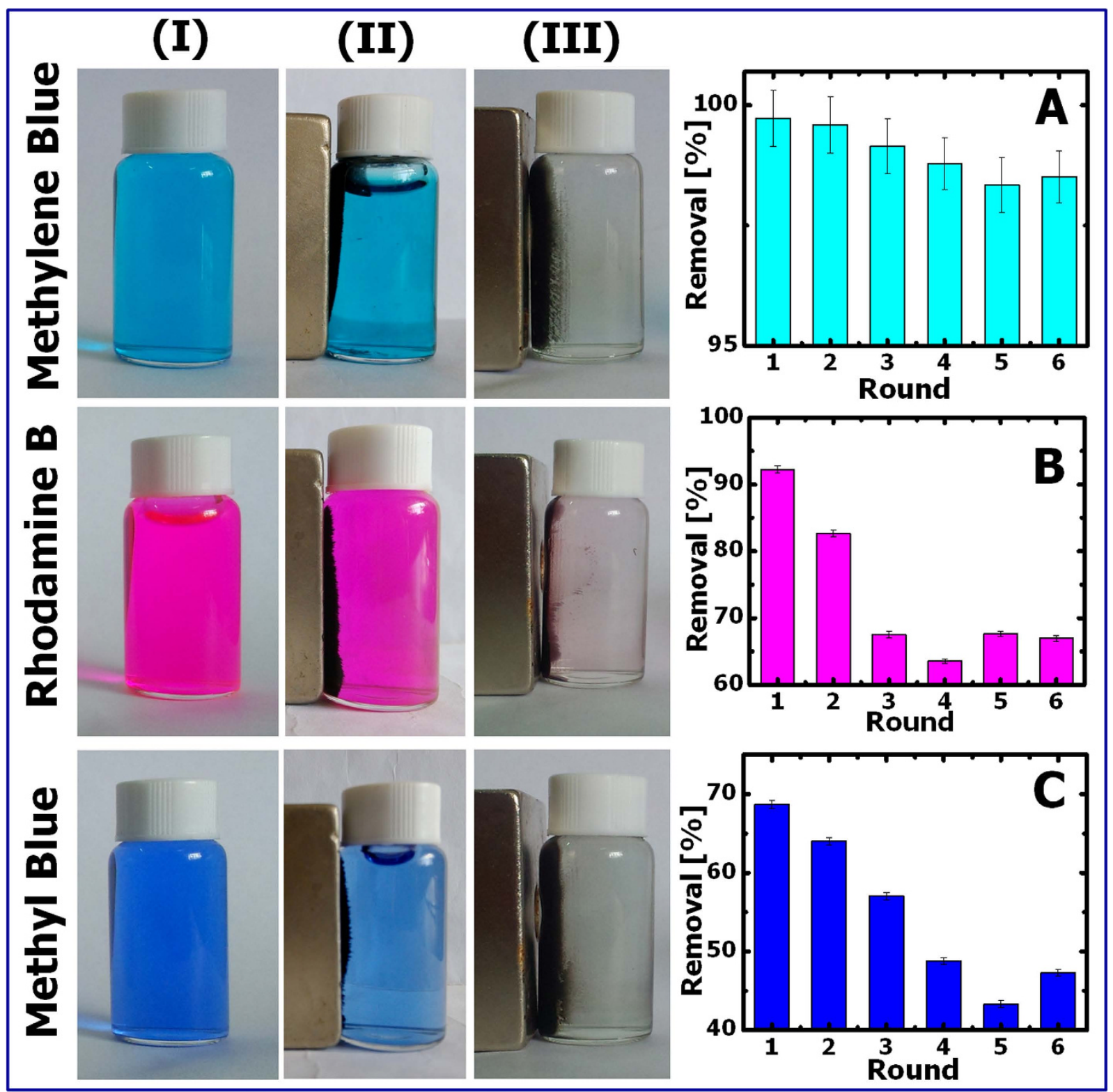

Figure 7. Photographs of dye solutions $\left(20 \mathrm{mg} \cdot \mathrm{L}^{-1}\right)$ before (I) and after (II) adding $\mathrm{Fe}_{3} \mathrm{O}_{4}$ multifaceted nanoparticles or (III) adding $\mathrm{GO} / \mathrm{Fe}_{3} \mathrm{O}_{4}$ nanohybrids. Percent dye removal rate was determined after 12 hour adsorption with constant shaking for the as-prepared $\mathrm{GO} / \mathrm{Fe}_{3} \mathrm{O}_{4}$ nanohybrids (Round 1) and for the regenerated $\mathrm{GO} / \mathrm{Fe}_{3} \mathrm{O}_{4}$ nanohybrids (Round 2-6), as seen in (A) MLB, and (B) $\mathrm{RhB}$, and (C) MB.

Synthesis of $\mathrm{Fe}_{3} \mathrm{O}_{4}$ Nanoparticles. Various $\mathrm{Fe}_{3} \mathrm{O}_{4}$ nanoparticles were prepared by a modified solvothermal method ${ }^{13}$. Ferric chloride hexahydrate $(1.35 \mathrm{~g})$ was first dissolved in $30 \mathrm{~mL}$ ethylene glycol, followed by adding sodium hydroxide (e.g., $0.4 \mathrm{~g}, 0.6 \mathrm{~g}$, and $0.8 \mathrm{~g}$ ) and polyethylene glycol $(1.0 \mathrm{~g})$ into the solution mixtures. After stirring for $30 \mathrm{~min}$, the solution mixtures were transferred into a Teflon-lined stainless steel autoclave $(100 \mathrm{ml})$ and reacted at $200^{\circ} \mathrm{C}$ for $8 \mathrm{~h}$. After the reaction was complete, the black precipitate (i.e., the $\mathrm{Fe}_{3} \mathrm{O}_{4}$ nanoparticles) was collected using an external magnetic field, followed by alternately washing with deionized water and ethanol several times. Finally, the $\mathrm{Fe}_{3} \mathrm{O}_{4}$ nanoparticles were dried at $60^{\circ} \mathrm{C}$ in a vacuum oven.

Preparation of Sulfonated GO Sheets. The GO sheets were prepared using a modified Hummer's method ${ }^{9,10}$. Graphite, $\mathrm{NaNO}_{3}$, and concentrated $\mathrm{H}_{2} \mathrm{SO}_{4}$ were mixed together in a beaker in an ice bath for $30 \mathrm{~min}$, followed by the slow addition of $\mathrm{KMnO}_{4}$. The reaction mixture was stirred at $35^{\circ} \mathrm{C}$ for $6 \mathrm{~h}$, and then the temperature was slowly raised to $60^{\circ} \mathrm{C}$ during the next $2 \mathrm{~h}$. The above mixture was then added to water and was stirred at $80^{\circ} \mathrm{C}$ for $1 \mathrm{~h}$, followed by adding $30 \% \mathrm{H}_{2} \mathrm{O}_{2}$ and filtering. For purification, the product was alternately washed with $5 \%$ of $\mathrm{HCl}$ and then $\mathrm{DI} \mathrm{H}_{2} \mathrm{O}$ several times. The filter cake was dissolved in $\mathrm{DI} \mathrm{H}_{2} \mathrm{O}$ and the graphene oxide flakes were obtained through centrifugation. Finally, the 
product was freeze-dried in a lyophilizer for 2 days. In order to increase the capacity of uploading $\mathrm{Fe}_{3} \mathrm{O}_{4}$ nanoparticles, the GO sheets were sulfonated using a published method ${ }^{12}$. The prepared GO suspension $(40 \mathrm{~mL})$ was sulfonated with $98 \%$ sulfuric acid $(10 \mathrm{~mL})$ in an ice bath for $2 \mathrm{~h}$, and washed with DI $\mathrm{H}_{2} \mathrm{O}$ several times to remove the remaining acid. The sulfonated GO sheets thus prepared were dried using a lyophilizer.

Preparation of sulfonated $\mathrm{GO} / \mathrm{Fe}_{3} \mathrm{O}_{4}$ Nanoparticle Hybrids. The sulfonated GO dispersion (50 mg GO in $40 \mathrm{~mL}$ water) was prepared by ultrasonicating GO in water for $1 \mathrm{~h}$. A given amount of the $\mathrm{Fe}_{3} \mathrm{O}_{4}$ nanoparticles (e.g., $20 \mathrm{mg}$ and $40 \mathrm{mg}$ ) was added to the $\mathrm{GO}$ aqueous dispersion. The mixture was then vigorously stirred for $30 \mathrm{~min}$ and ultrasonicated for $2 \mathrm{~h}$ to achieve a homogeneous suspension. The $\mathrm{GO} / \mathrm{Fe}_{3} \mathrm{O}_{4}$ hybrids were collected by centrifuging and applying an external magnetic field to removeany floating $\mathrm{GO}$ sheets. Finally, $\mathrm{GO} / \mathrm{Fe}_{3} \mathrm{O}_{4}$ hybrids were alternately washed with ethanol and $\mathrm{DI} \mathrm{H}_{2} \mathrm{O}$ several times and dried using a lyophilizer.

Dye Adsorption Experiments. The adsorption of dyes on the hybrid materials was examined using absorption spectroscopy. The prepared nanocomposites $(3 \mathrm{mg})$ were added into a dye solution $(20 \mathrm{mg}$ $\mathrm{L}^{-1}, 5 \mathrm{~mL}$ ). The mixtures were ultrasonicated for 3 minutes and kept on a shaking table at $25^{\circ} \mathrm{C}$. The concentrations of residual dye in the aqueous solution were tested at different time intervals. The $\mathrm{pH}$ values of dye solutions were all adjusted to 6 . This value was selected by optimizing the $\mathrm{pH}$ value for $\mathrm{RhB}$ (see Fig S8). A temperature of $25^{\circ} \mathrm{C}$ and a $\mathrm{pH}$ value of 6 were then used for all absorption tests. Absorption values for a series of dye solutions with known concentrations were measured at the maximum absorption wavelength using a UV-vis spectrophotometer $(554 \mathrm{~nm}$ for Rhodamine B, $664 \mathrm{~nm}$ for Methylene Blue, and $600 \mathrm{~nm}$ for Methyl Blue). Three calibration curves were therefore constructed in order to determine residual dye concentrations during adsorption experiments. The absorption value of the residual dyes was also determined at the maximum absorption wavelength using a UV-vis spectrophotometer.

The Regeneration of the Hybrid Adsorbents. Once saturated adsorption for a given dye in an aqueous suspension was reached after $12 \mathrm{~h}$ on a shaking table at $25^{\circ} \mathrm{C}$, the hybrid was extracted from solution through an external magnetic attraction, and washed alternately using ethylene glycol and eth-

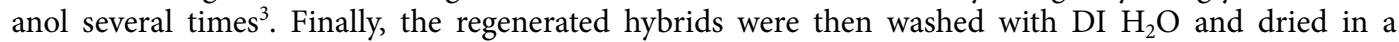
lyophilizer for subsequent adsorption experiments.

Characterization. Scanning electron microscope (SEM) images were taken using a Hitachi S-4800 field emission scanning electron microscope (Chiyoda-ku, Japan) with an accelerating voltage of 5-15kV. All transmission electron microscope (TEM) measurements were carried out using a Hitachi HT7700 transmission electron microscope (Hitachi, Tokyo, Japan). The X-ray diffraction (XRD) measurement was conducted using a Rigaku D/max 2550PC diffractometer (Rigaku Inc., Tokyo, Japan). The XRD pattern was obtained using $\mathrm{CuK} \alpha$ radiation with an incident wavelength of $0.1542 \mathrm{~nm}$ under a voltage of $40 \mathrm{kV}$ and a current of $200 \mathrm{~mA}$. The scan rate was 0.5 degree $\cdot \mathrm{min}^{-1}$. The magnetic properties of the samples were tested by a vibrating sample magnetometer (VSM) of the Physical Property Measurement System (PPMS, Quantum design Model 6000). The powders were aligned under a $4 \mathrm{kOe}$ field and solidified with epoxy resin for the VSM measurements. Raman spectroscopy was performed using a Horiba Jobin Yvon Xplora PLUS confocal Raman microscope equipped with a motorized sample stage. The wavelength of the excitation laser was $532 \mathrm{~nm}$ and the power of the laser was kept below $1 \mathrm{~mW}$ without noticeable sample heating. The intensity of a Raman peak was extracted from the maximum value after baseline subtraction over corresponding spectral range.

\section{References}

1. Xue, Y. et al. Oxidizing metal ions with graphene oxide: the in situ formation of magnetic nanoparticles on self-reduced graphene sheets for multifunctional applications. Chem. Commun. 47, 11689-91 (2011)

2. Geng, Z. et al. Highly efficient dye adsorption and removal: a functional hybrid of reduced graphene oxide-Fe $\mathrm{O}_{4}$ nanoparticles as an easily regenerative adsorbent. J. Mater. Chem. 22, 3527-35 (2012).

3. Sun, H., Cao, L. \& Lu, L. Magnetite/reduced graphene oxide nanocomposites: One step solvothermal synthesis and use as a novel platform for removal of dye pollutants. Nano Research 4, 550-62 (2011).

4. Yang, X. et al. Graphene oxide-iron oxide and reduced graphene oxide-iron oxide hybrid materials for the removal of organic and inorganic pollutants. RSC Advances 2, 8821-6 (2012).

5. Meidanchi, A. \& Akhavan, O. Superparamagnetic zinc ferrite spinel-graphene nanostructures for fast wastewater purification. Carbon 69, 230-8 (2014)

6. Fan, X., Jiao, G., Zhao, W., Jin, P. \& Li, X. Magnetic $\mathrm{Fe}_{3} \mathrm{O}_{4}$-graphene composites as targeted drug nanocarriers for pH-activated release. Nanoscale 5, 1143-52 (2013).

7. Sharma, P. \& Das, M. R. Removal of a Cationic Dye from Aqueous Solution Using Graphene Oxide Nanosheets: Investigation of Adsorption Parameters. J. Chem. En. Data 58, 151-8 (2013).

8. Zhang, Y., Yuan, S., Zhao, Y., Wang, H. \& He, C. Synthesis of novel yttrium-doped graphene oxide nanocomposite for dye removal. J. Mater. Chem. A 2, 7897-903 (2014).

9. Hummers Jr., W. S. \& Offeman, R. E. Preparation of graphitic oxide. J. Am. Chem. Soc. 80, 1339-9 (1958).

10. Li, D., Muller, M. B., Gilje, S., Kaner, R. B. \& Wallace, G. G. Processable aqueous dispersions of graphene nanosheets. Nat Nanotechnol 3, 101-5 (2008).

11. Ravikumar \& Scott, K. Freestanding sulfonated graphene oxide paper: a new polymer electrolyte for polymer electrolyte fuel cells. Chem. Commun. 48, 5584-6 (2012). 
12. Liu, J., Xue, Y. \& Dai, L. Sulfated Graphene Oxide as a Hole-Extraction Layer in High-Performance Polymer Solar Cells. J. Phys. Chem. Lett. 3, 1928-33 (2012).

13. Han, Q. et al. Facile and tunable fabrication of $\mathrm{Fe} 3 \mathrm{O}$ 4/graphene oxide nanocomposites and their application in the magnetic solid-phase extraction of polycyclic aromatic hydrocarbons from environmental water samples. Talanta 101, 388-95 (2012).

14. Du, X., Guo, P., Song, H. H. \& Chen, X. H. Graphene nanosheets as electrode material for electric double-layer capacitors. Electrochim. Acta 55, 4812-4819 (2010).

15. Ferrari, A. C. \& Robertson, J. Interpretation of Raman spectra of disordered and amorphous carbon. Phys. Rev. B 61, 14095-14107 (2000).

16. Malard, L. M., Pimenta, M. A., Dresselhaus, G. \& Dresselhaus, M. S. Raman spectroscopy in graphene. Phys. Rep. 473, 51-87 (2009)

17. Calizo, I., Balandin, A. A., Bao, W., Miao, F. \& Lau, C. N. Temperature dependence of the raman spectra of graphene and graphene multilayers. Nano Lett. 7, 2645-2649 (2007).

18. Kudin, K. N. et al. Raman spectra of graphite oxide and functionalized graphene sheets. Nano Lett. 8, 36-41 (2008).

19. Akhavan, O. Bacteriorhodopsin as a superior substitute for hydrazine in chemical reduction of single-layer graphene oxide sheets. Carbon 81, 158-166 (2015).

20. Akhavan, O. \& Ghaderi, E. Graphene nanomesh promises extremely efficient in vivo photothermal therapy. Small 9, 3593-3601 (2013).

21. Balashov, T., Takács, A. F., Wulfhekel, W. \& Kirschner, J. Magnon excitation with spin-polarized scanning tunneling microscopy. Phys. Rev. Lett. 97, 187201 (2006).

22. Kim K. S. et al. Large-scale pattern growth of graphene films for stretchable transparent electrodes. Nature 457, 706-710 (2009).

23. Kan, J. \& Wang, Y. Large and fast reversible Li-ion storage in Fe2O3-graphene sheet-on-sheet sandwich-like nanocomposites. Sci Rep. 3, 3502; doi: 10.1038/srep03502 (2013).

24. Zubir, A., Yacou, C., Motuzas, J., Zhang, X. \& Costa, J. C. D. Structural and functional investigation of graphene oxide-Fe 3 O 4 nanocomposites for the heterogeneous Fenton-like reaction. Sci Rep. 4, 4594; doi:10.1038/srep04594 (2014).

25. Chidembo, A. et al. Globular reduced graphene oxide-metal oxide structures for energy storage applications. Energ. Environ. Sci. 5, 5236-40 (2012).

26. Qian, W. et al. Surfactant-free hybridization of transition metal oxide nanoparticles with conductive graphene for highperformance supercapacitor. Green Chem. 14, 371-377 (2012).

27. Barman, B. K. \& Nanda, K. K. Si-mediated fabrication of reduced graphene oxide and its hybrids for electrode materials. Green Chem. 17, 776-80 (2015)

28. Zhang, Q. et al. Rationally designed porous polystyrene encapsulated zirconium phosphate nanocomposite for highly efficient fluoride uptake in waters. Sci Rep 3, 2551; doi: 10.1038/srep02551 (2013).

29. Zhao, X. et al. Selective anion exchange with nanogated isoreticular positive metal-organic frameworks. Nat Commun 4, 2344; doi: $10.1038 /$ ncomms3344 (2013).

30. Canning, J. et al. Percolation Diffusion into Self-Assembled Mesoporous Silica Microfibres. Nanomaterials 4, 157-74 (2014)

\section{Acknowledgments}

National Natural Science Foundation of China (21473153 and 21207112), TJ, QZ; Natural Science Foundation of Hebei Province (No. B2013203108), TJ; Science Foundation for the Excellent Youth Scholars from Universities and Colleges of Hebei Province (Nos. Y2011113 and YQ2013026), QZ, TJ; Support Program for the Top Young Talents of Hebei Province, TJ; Open Foundation of National Key Laboratory of Biochemical Engineering (Institute of Process Engineering of Chinese Academy of Sciences), TJ; CMU Provost Discretionary Award, BLI. The funders had no role in study design, data collection and analysis, decision to publish, or preparation of the manuscript.

\section{Author Contributions}

The research was planned by T.F.J., Y.W. and Y.L. Experiments were performed by Y.L., T.J. and Y.W. supervised the research. T.J. and B.L.I. prepared the manuscript. Q.Z., X.Y., F.G., A.B., T.Z. and J.L. contributed with discussion, feedback, and comments on the manuscript.

\section{Additional Information}

Supplementary information accompanies this paper at http://www.nature.com/srep

Competing financial interests: The authors declare no competing financial interests.

How to cite this article: Jiao, T. et al. Facile and Scalable Preparation of Graphene Oxide-Based Magnetic Hybrids for Fast and Highly Efficient Removal of Organic Dyes. Sci. Rep. 5, 12451; doi: 10.1038/srep12451 (2015).

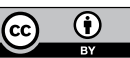

This work is licensed under a Creative Commons Attribution 4.0 International License. The images or other third party material in this article are included in the article's Creative Commons license, unless indicated otherwise in the credit line; if the material is not included under the Creative Commons license, users will need to obtain permission from the license holder to reproduce the material. To view a copy of this license, visit http://creativecommons.org/licenses/by/4.0/ 